\title{
Review Article \\ Roles of Cells from the Arterial Vessel Wall in Atherosclerosis
}

\author{
Di Wang, Zhiyan Wang, Lili Zhang, and Yi Wang \\ Department of Cardiology, Shanghai General Hospital, School of Medicine, Shanghai Jiao Tong University, Shanghai, China
}

Correspondence should be addressed to Yi Wang; wangyipublic@hotmail.com

Received 10 January 2017; Revised 26 April 2017; Accepted 2 May 2017; Published 7 June 2017

Academic Editor: Fumio Tsuji

Copyright @ 2017 Di Wang et al. This is an open access article distributed under the Creative Commons Attribution License, which permits unrestricted use, distribution, and reproduction in any medium, provided the original work is properly cited.

\begin{abstract}
Atherosclerosis has been identified as a chronic inflammatory disease of the arterial vessel wall. Accumulating evidence indicates that different cells from the tunica intima, media, adventitia, and perivascular adipose tissue not only comprise the intact and normal arterial vessel wall but also participate all in the inflammatory response of atherosclerosis via multiple intricate pathways. For instance, endothelial dysfunction has historically been considered to be the initiator of the development of atherosclerosis. The migration and proliferation of smooth muscle cells also play a pivotal role in the progression of atherosclerosis. Additionally, the fibroblasts from the adventitia and adipocytes from perivascular adipose tissue have received considerable attention given their special functions that contribute to atherosclerosis. In addition, numerous types of cytokines produced by different cells from the arterial vessel wall, including endothelium-derived relaxing factors, endothelium-derived contracting factors, tumor necrosis factors, interleukin, adhesion molecules, interferon, and adventitium-derived relaxing factors, have been implicated in atherosclerosis. Herein, we summarize the possible roles of different cells from the entire arterial vessel wall in the pathogenesis of atherosclerosis.
\end{abstract}

\section{Introduction}

As a major public health issue, atherosclerosis in concert with its related disorders, such as coronary heart diseases, stroke, and peripheral vascular diseases, has been the leading cause of mortality and morbidity worldwide $[1,2]$. In the recent years, some of the pioneers have unceasingly devoted themselves to investigate the possible mechanisms implicated in the pathogenesis of atherosclerosis and have made considerable progress, such as the "response to inflammation" theory based on "response to injury" theory [3] and "response to lipoprotein retention" hypothesis [4-6]. In recent years, the concept that atherosclerosis is a chronic inflammatory disease has been extensively accepted [7-10]. In contrast, uncertainty surrounds whether vascular inflammation is transmitted as traditionally thought via "inside to outside" responses emphasizing the indispensable roles of the intima in the progression of atherosclerosis. Alternatively, the new paradigm of an "outside to inside" hypothesis is supported by compelling evidence $[11,12]$, which predominantly uncovers the functional significance of the tunica intima, adventitia, and perivascular adipose tissue (also called perivascular fat, PVAT).

Additionally, classical doctrine indicates that smooth muscle cells (SMCs) predominantly from the medial have a crucial impact on the development of atherosclerosis based on their migration into the intima and proliferation [13]. Thus, this review will shed light on different cells, including endothelial cells (ECs), SMCs, fibroblasts, and adipocytes from the tunica intima, media, adventitia, and PVAT and their related cytokines, and elucidate how these cells contribute to the pathogenesis of atherosclerosis (Figure 1).

\section{The Normal Structure of the Arterial Vessel Wall}

To the best of our knowledge, some of the large and moderate arteries, such as the aorta and coronary artery, are composed of the tunica intima, the media, and the adventitia encapsulated by perivascular adipose tissue. Longitudinal vascular ECs covering the inner surface of blood vessels are crucial components of the tunica intima and are sequentially 


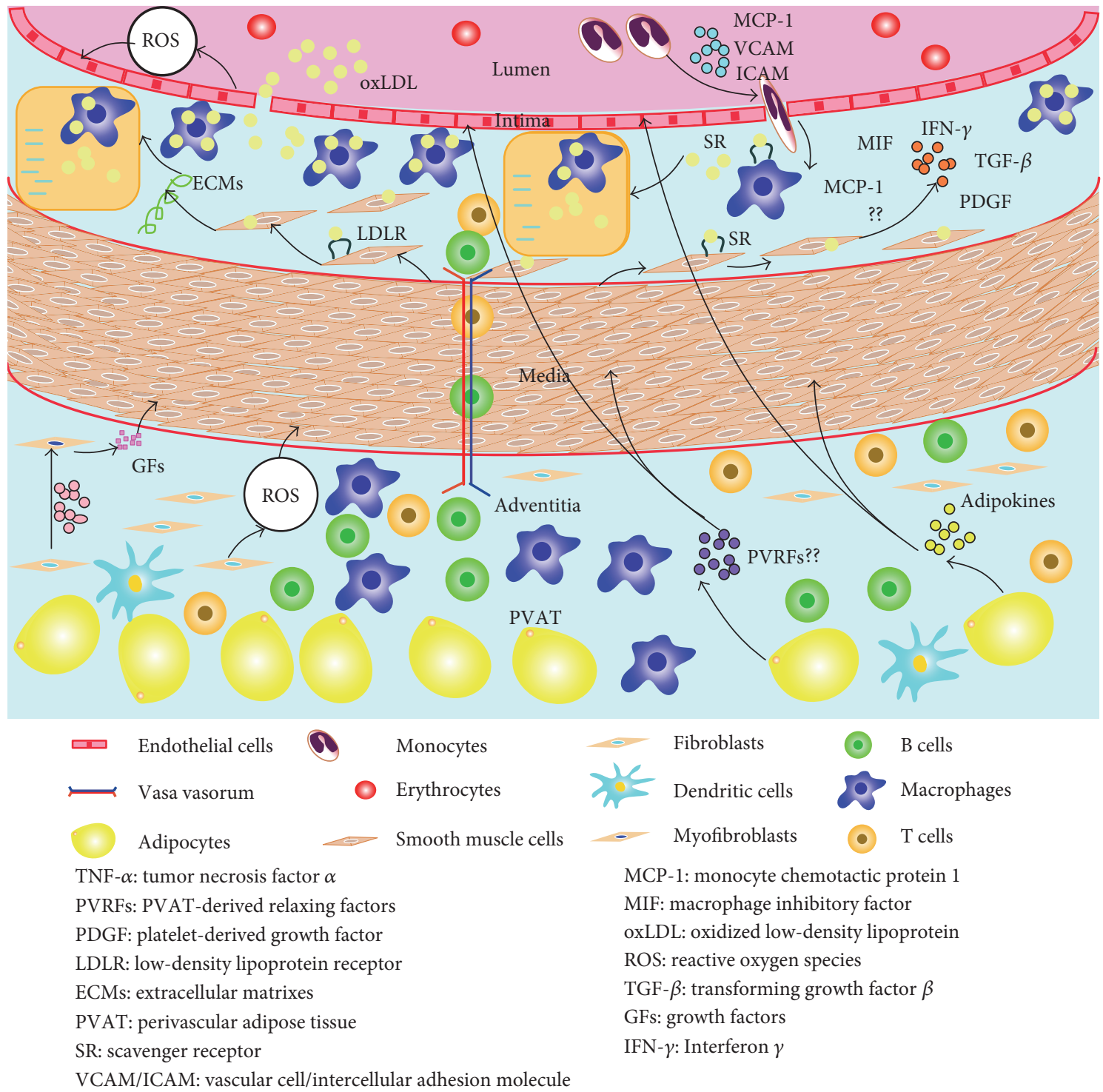

FIGURE 1: Roles of different cells from the arterial vessel wall in atherosclerosis. Different cells, including endothelial cells, smooth muscle cells, fibroblasts, and adipocytes from the tunica intima, media, adventitia, and perivascular adipose tissue and their related cytokines all participate in the inflammatory response of atherosclerosis via multiple intricate pathways. Endothelial dysfunction, smooth muscle cell migration and proliferation, the transformation of fibroblasts into myofibroblasts, and adipokines produced by perivascular adipose tissue are predominantly implicated in the pathological process of atherosclerosis.

exposed to shear stress due to frictional force from the blood flow [14]. The tunica media is saturated with elastic fibers secreted by sparse SMCs in large arteries (also called elastic arteries); nevertheless, the media of moderate arteries (also named muscular arteries) is circumferentially composed of smooth muscle cells side by side together with disseminated elastic fibers and collagen fibers. As the outermost layer of arterial vessel wall, the adventitia consists of fibroblasts, vasa vasorum, nerve endings, and a few resident inflammatory cells in the loose connective tissue [15]. PVAT is located in the outside of the adventitia without any organized barrier to insulate the two, mainly encompassing adipocytes and other infiltrating immune cells, such as macrophages, $\mathrm{T}$ cells, fibroblasts, and capillary endothelial cells, as reviewed by Szasz and Webb [16] (Figure 2).

\section{The Intima in the Development of Atherosclerosis}

3.1. The Normal Functions of the Intima. As the endocrinal "organ" of the cardiovascular system, ECs mainly consist of the intima with extremely activated metabolism and exceedingly complicated functions. For example, ECs secrete numerous bioactive substances to modulate vascular tone, such as nitric oxide $(\mathrm{NO})$, prostaglandin $\mathrm{I}_{2}\left(\mathrm{PGI}_{2}\right)$, and endothelium-derived hyperpolarizing factor (EDHF), which are regarded as endothelium-derived relaxing factors 


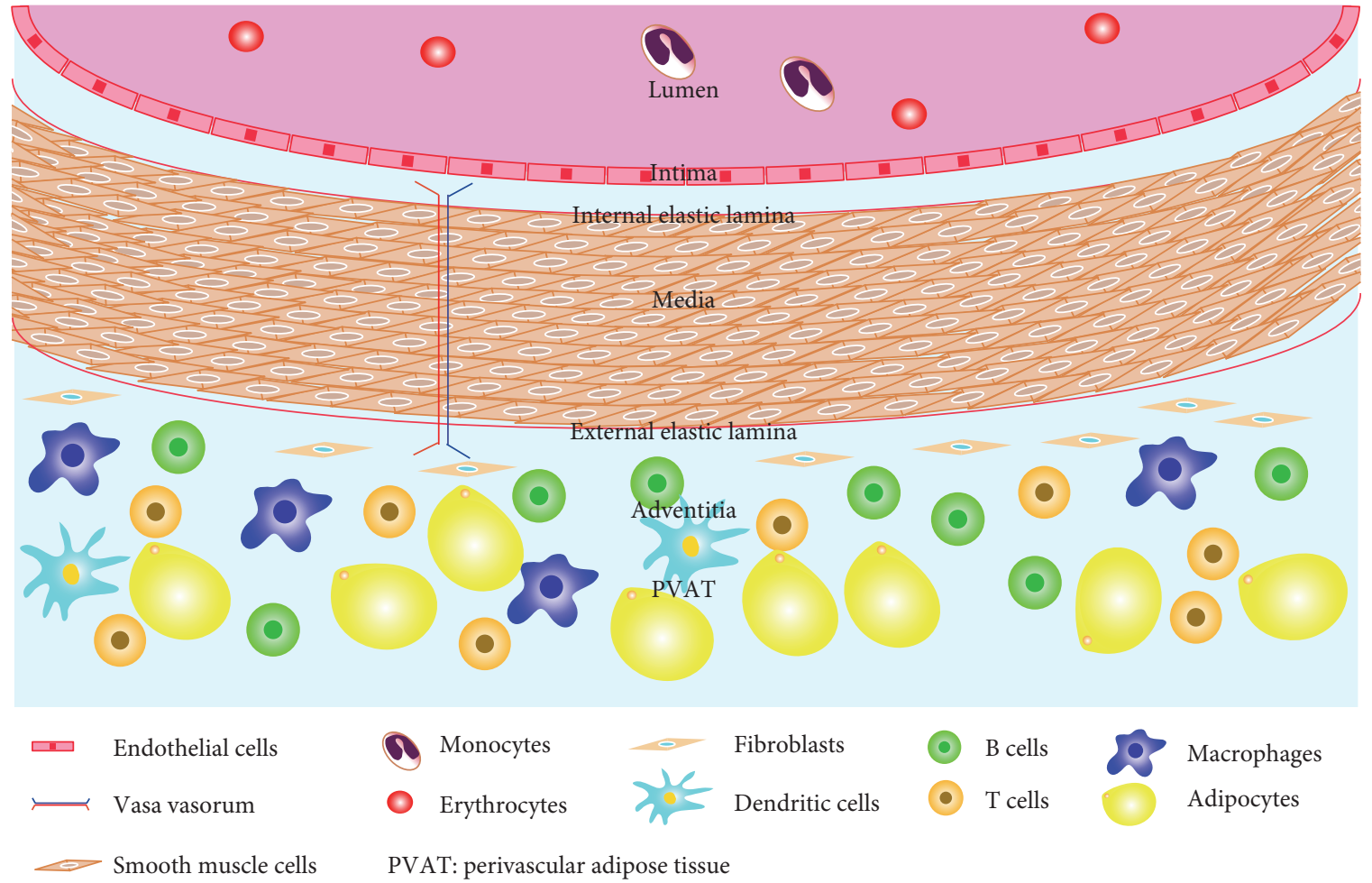

Figure 2: The normal structure of the arterial vessel wall. The intima, media, adventitia, and perivascular adipose tissue comprise the intact and normal arterial vessel wall. Therefore, cells from the arterial vessel wall mainly consist of endothelial cells, smooth muscle cells, fibroblasts, and adipocytes. Other cells, such as macrophages, dendritic cells, and T cells, also reside in the arterial vessel wall. In addition, the vasa vasorum promotes blood and oxygen delivery to the arterial vessel wall.

(EDRFs) as well as endothelin 1 (ET-1), thromboxane $A_{2}$ $\left(\mathrm{TXA}_{2}\right)$, angiotensin II (Ang II), and uridine adenosine tetraphosphate $\left(\mathrm{UP}_{4} \mathrm{~A}\right)$, which belong to endothelium-derived contracting factors (EDCFs). On the one hand, ECs are natural barriers of the blood vessel to maintain the smoothness of the tunica intima to prevent the platelet and leukocyte adhesion and hazardous molecules from invading into arterial vessel wall. On the other hand, the intact endothelium maintains a physiological equilibrium related to the processes of thrombosis via releasing antithrombotic and thrombotic substances and vascular smooth muscle proliferation by constituting the basement membrane of collagen together with the protective layer of SMCs [17]. Interestingly, ECs exert an important effect on the exchange of substances and active transport [18].

3.2. Roles of Endothelial Cells in Atherosclerosis. Endothelial dysfunction (ED) is the primary and a crucial step of the development of atherosclerosis. Numerous cardiovascular risk factors, such as obesity and diabetes mellitus, potentially initiate endothelial cell injury, causing ED [19]. Under normal conditions, the endothelium regulates vascular inflammation by secreting NO, whereas a dysfunctional endothelium accelerates reactive oxygen species (ROS) generation and increases vascular inflammation, which is harmful to the vascular system [20]. The damage to the endothelium upsets the balance between vasoconstriction and vasodilation, which is characterized by increased EDCFs, especially ET-1, and reduced EDRFs, mainly NO, and initiates a series of pathophysiologic changes that promote or exacerbate atherosclerosis, including increased vascular permeability to lipoproteins and augmented leukocyte adhesion, platelet aggregation, and generation of cytokines [7]. On the other hand, various inflammatory cytokines, such as tumor necrosis factor $\alpha$ (TNF- $\alpha$ ), interleukin 1 (IL-1), and IL-6, induce the endothelium to express vascular cell adhesion molecule (VCAM), intercellular adhesion molecule (ICAM), monocyte chemoattractant protein 1 (MCP-1), and other chemokines, consequently promoting the adherence and migration of monocytes [21-24]. Once resident in the intima, monocytes acquire characteristics of tissue macrophages. Monocytes augment the expression of scavenger receptor (SR) and then internalize modified lipoproteins. The above processes consequently lead to the formation of foam cells (FCs), which can be regarded as the early atherosclerotic lesion [8]. After the rupture of the atherosclerotic plaque, the physiological balance between antithrombotic and thrombotic substances is disrupted due to the dysfunction of ECs, which leads to increased thrombotic substances (e.g., von Willebrand factor $(\mathrm{vWF}), \mathrm{TXA}_{2}$ ) and attenuated antithrombotic substances, such as heparin. These effects facilitate the process of thrombosis, causing devastating consequences [25]. In conclusion, all the above-mentioned factors contribute to atherosclerosis, indicating the indispensable roles of endothelial cells in the progression of atherosclerosis. 


\section{The Media in the Progression of Atherosclerosis}

4.1. The Normal Roles of the Media. As mentioned previously, the thickness and components of the media between the tunica intima and adventitia depend on the artery type. The elastic fibers characterized by extensibility, mainly forming the media of large arteries, maintain the intact structure and elastic contraction of arteries. However, the media of moderate arteries is circumferentially saturated with smooth muscle cells in a side-by-side arrangement. Owens recommended that the fully differentiated or mature SMCs could express a unique repertoire of contractile proteins (e.g., smooth muscle myosin heavy chain (SM-MHC) or $\alpha$ smooth muscle actin ( $\alpha \mathrm{SMA})$ ), ion channels, and signaling molecules that are required for its contractile function [26]. During vasculogenesis, SMCs also produce high levels of extracellular matrices (ECMs), including collagen, elastin, proteoglycans, cadherins, and integrins that consist of a major portion of the blood vessel mass [27]. In addition, other components of media collagen fibers play a crucial role in connecting and supporting the blood vessel.

4.2. Smooth Muscle Cells Contributing to Atherosclerosis. The relationship between SMCs and atherosclerosis has considerably drawn attention since Ross et al. proposed that SMCs have a principal impact on the development of atherosclerosis due to their migration into the intima and proliferation [13]. Interestingly, migrated SMCs are key players in the process of luminal stenosis after the damage of the intima and the internal elastic lamina. Once numerous SMCs migrate to the intima, their excessive proliferation and apoptosis suppression promote extracellular matrix synthesis and lipid deposition, consequently facilitating arterial wall fibrosis and thickening and the luminal stenosis. First, Ruan et al. and Ishikawa et al. demonstrated that human SMCs express numerous lipid uptake receptors, such as lowdensity lipoprotein receptor (LDLR) and SR, contributing to the formation of myogenic FCs [28, 29]. Second, SMC proliferation can be inhibited by NO, which is a key component of arterial vessel wall remodeling in response to injury, for example, after angioplasty or vein grafting and during atherosclerosis formation [30]. Hou et al. provided new evidence that vasostatin-2 may function as an atherosclerosis-related factor that inhibits cell proliferation and cell adhesion in SMCs, which are associated with the progression of atherosclerosis [31]. In addition, Lang et al. strongly suggested that luteolin suppresses the migration and proliferation of SMCs via downregulating Akt and Src signals [32]. As mentioned above, the migration and proliferation of SMCs are an indispensable pathological process of atherosclerosis. In addition, some of the ECMs released by SMCs strengthen the fibrous cap of the atherosclerotic plaque to protect against plaque rupture and thrombosis [33]. Shankman et al. demonstrated that the contribution of SMCs within atherosclerotic plaques has been extremely neglected and that the transformation of the SMC phenotype mediated by KLF4 is crucial in lesion pathogenesis utilizing a Myh11CreERT2 ROSA floxed STOP eYFP ApoE ${ }^{-/-}$mouse model
[34]. In addition, a comprehensive review by Doran et al. listed numerous cytokines likely produced by SMCs, such as platelet-derived growth factor (PDGF), transforming growth factor $\beta$ (TGF- $\beta$ ), macrophage inhibitory factor (MIF), interferon $\gamma$ (IFN- $\gamma$ ), and MCP-1. These cytokines are likely derived from other cells within lesion cells; thus, the concrete functions of these cytokines in the progression of atherosclerosis remain unclear [35].

\section{The Adventitia Contributing to Atherosclerosis}

Recently, the adventitia, which is the outermost layer of arterial vessel wall, has attracted considerable interest given its complex and dynamic roles. Traditionally, the adventitia was regarded as merely an inert physical barrier separating tissues to provide support for blood vessels and a scaffold for the sympathetic nerve system and the vasa vasorum [36]. However, compelling evidence demonstrated that the adventitia plays a critical role in coordinating the progression of atherosclerosis. In 1962, Schwartz and Mitchell demonstrated that the prevalence and degree of the adventitial cellular infiltration closely correlated with the severity of the atherosclerotic plaque [37]. The most common fibroblasts in the adventitia have the capacity of differentiating into myofibroblasts mainly activated by TGF- $\beta$ [38], consequently increasing local expression of inflammatory cytokines and growth factors (GFs) [39, 40]. In addition, Xu et al. demonstrated that the earliest expression of MCP-1 was detected in the adventitial fibroblasts before the formation of intimal lesions after feeding $\mathrm{ApoE}^{-1-}$ mice a hyperlipidic diet [41]. On the other hand, adventitial fibroblast nicotinamide adenine dinucleotide phosphate hydrate (NADPH) oxidase-derived ROS is the sensor and messenger for the early development of vascular disease [42]. For example, Liu et al. recommended that NADPH oxidase inhibitors reduced vascular ROS and the medial area via delivering the $\mathrm{NADPH}$ oxidase inhibitor gene to the vascular adventitia in C57BL/6 mice [43]. In addition, after the continuous hyperlipidic diet administration, the activated fibroblasts (AFs) derived from $\mathrm{ApoE}^{-/-}$mice displayed augmented NADPH oxidase activity, $\mathrm{O}^{-}$production, and increased $\mathrm{p} 47 \mathrm{phox}$ levels compared with wild-type mice. These effects are associated with increased proliferation and migration of AFs. In addition, p47phox knockout mediated by siRNA decreased the proliferation and migration of $\mathrm{AFs}$ in $\mathrm{ApoE}^{-/-}$mice [44]. Additionally, the vasa vasorum promotes blood and oxygen delivery to the arterial vessel wall, thus providing a suitable environment for the development of atherosclerotic plaque and serving as a conduit for trafficking of resident and progenitor cells into the media and intima [45]. Additionally, $\mathrm{Hu}$ et al. hypothesized that adventitial transient receptor potential vanilloid type 1 (TRPV1) and sensory Cfibers may play a pivotal role in the adventitia, underscoring the role of the sympathetic nerve system in the development of atherosclerosis [46].

In addition to fibroblasts, some of the lymphocytes accumulate in the adventitia, as supported by the study that demonstrated that the adventitia is a major site of arterial wall 


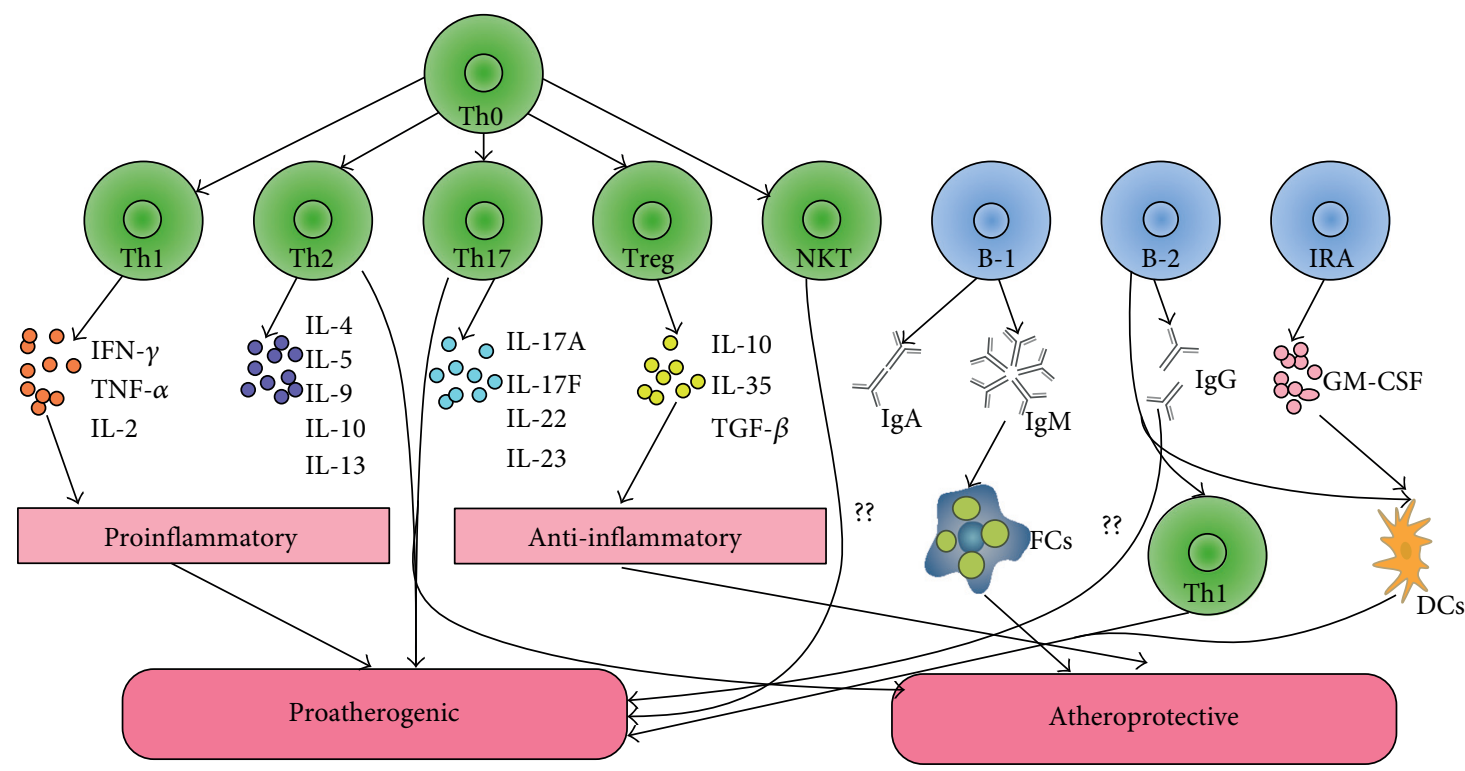

Th: T helper cells

IFN- $\gamma$ : interferon $\gamma$

GM-CSF: granulocyte-macrophage colony-stimulating factor
TNF- $\alpha$ : tumor necrosis factor $\alpha$

Treg: regulatory $\mathrm{T}$ cells

FCs: foam cells

NKT: natural killer T cells
DCs: dendritic cells

TGF- $\beta$ : transforming growth factor $\beta$

IRA: innate response activator cells

IL: interleukin

FIGURE 3: The effect of T cell or B cell subsets in atherosclerosis. Th0 cells can differentiate into Th1, Th2, Th17, Treg, and NKT cells. Th1 cells are proatherogenic cells that secrete proinflammatory cytokines. NKT cells are also proatherogenic cells, but the mechanism remains unclear. B-2 cells and IRA B cells are both proatherogenic cells, whereas B-1 B cells and Treg cells exert antiatherogenic effects. The effects of Th2 cells and Th17 cells in atherosclerosis remain unclear.

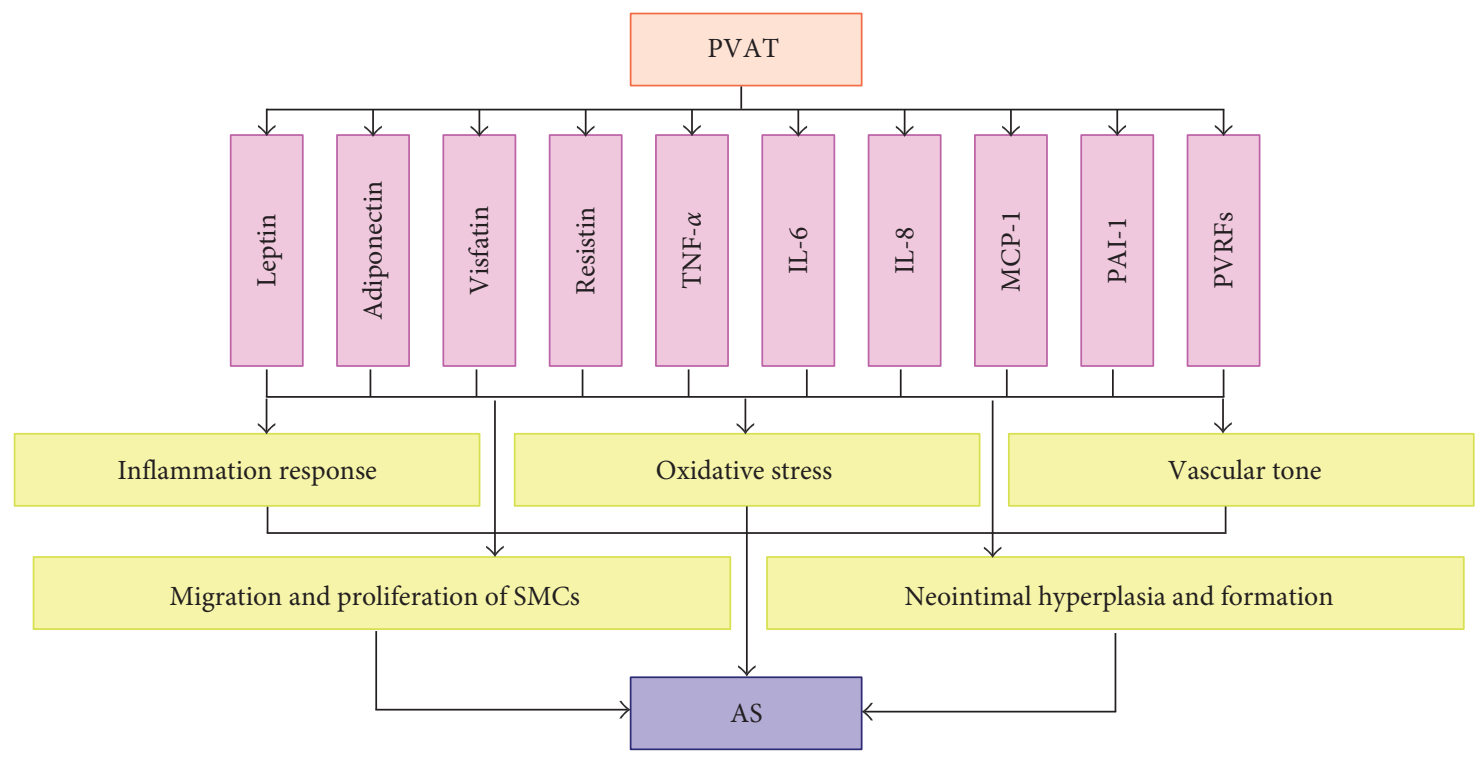

PVAT: perivascular adipose tissue

TNF- $\alpha$ : tumor necrosis factor $\alpha$

AS: atherosclerosis

IL-6: interleukin 6
MCP-1: monocyte chemotactic protein 1 PAI-1: plasminogen activitor inhibitor 1 PVRFs: PVAT-derived relaxing factors IL-8: interleukin 8

FIgURE 4: Perivascular adipose tissue contributing to atherosclerosis. PVAT plays a pivotal role in atherosclerosis by releasing numerous adipokines, such as leptin, adiponectin, visfatin, resistin, TNF- $\alpha$, IL-6, IL-8, MCP-1, and PAI-1. These adipokines mediate SMC migration and proliferation, also promoting neointimal hyperplasia and formation, stimulating inflammation responses and oxidative stress, and regulating vascular tone, consequently contributing to atherosclerosis. 
inflammation related to lymphocyte infiltration in atherosclerotic arteries [47]. T helper 1 (Th1) cells are proatherogenic cells that secrete proinflammatory cytokines, such as IL- 2 , TNF- $\alpha$, and IFN- $\gamma$. In contrast, regulatory T (Treg) cells are atheroprotective cells that release anti-inflammatory cytokines (e.g., IL-4, IL-5, IL-9, IL-10, and IL-13). Th2 cells and Th17 cells are proatherogenic cells and atheroprotective cells. Natural killer T (NKT) cells are proatherogenic cells; however, the mechanism remains unclear. B-1 cells exert antiatherogenic activities via secreting IgM, contributing to the formation of FCs. B-2 cells stimulate Th1 cells and dendritic cells (DCs) to play a proatherogenic role. B-2 cells also secrete $\operatorname{IgG}$, but its proatherogenic role remains to be elucidated. Innate responsive activator (IRA) cells exert proatherogenic activities by releasing granulocytemacrophage colony-stimulating factor (GM-CSF), which acts on DCs [48] (Figure 3).

\section{Multiple Roles of Perivascular Adipose Tissue in Atherosclerosis}

PVAT is defined as the adipose tissue around the arteries regardless of location [12] located on the outside of adventitia without laminar structures or any organized barrier to separate the two [16]. The old paradigm suggested that PVAT was merely a mechanical and structural support tissue for the blood vessel. More recently, we realized that PVAT not only stores triacylglycerols/triglycerides and free fatty acids (FFAs) participating in energy metabolism but also secretes quantities of adipokines, such as leptin, adiponectin, visfatin, resistin, TNF- $\alpha$, IL- 6 , IL- 8 , MCP-1, and plasminogen activator inhibitor 1 (PAI-1), which play indispensable roles in atherosclerosis by mediating SMC migration and proliferation [49], promoting neointimal hyperplasia and formation [50, 51], stimulating inflammation responses and oxidative stress [52], and regulating vascular tone [53] (Figure 4). For instance, Lamers et al. demonstrated that lipid mediators, such as FFAs and adipokines, affect SMC function via inducing augmented proliferation and inflammatory signaling and proposed that the increased fatty acids and adipokines released by PVAT in obesity may disclose the relationships among SMCs dysfunction, vascular inflammation, and atherosclerosis [54]. Additionally, PVAT plays an indispensable role in the inflammatory response to atherosclerosis. The result of proteomic analysis indicated that empirical adipose tissue (EAT) exhibits increased oxidative stress compared with subcutaneous adipose tissue (SAT) in patients with cardiovascular disease, suggesting its possible connection with myocardial stress. Similarly, perivascular visceral fat results in endothelial dysfunction and accelerates atherosclerosis as demonstrated by transplantation of visceral adipose tissue or SAT immediately adjacent to the right common carotid artery in $\mathrm{ApoE}^{-/-}$mice [55]. On the other hand, the idea that elevated levels of leptin may promote neointimal formation was observed in leptin-deficient ob/ob mice with reduced neointimal formation. In addition, Takaoka et al. found that endovascular injury significantly augments proinflammatory adipokines and attenuates adiponectin in a femoral artery wire injury mouse and iliac artery balloon injury rat. In addition, neointimal hyperplasia after vascular injury was reduced via knockout of TNF- $\alpha$ with decreased upregulation of proinflammatory adipokines. Regarding the regulation of vascular tone, adventitium-derived relaxing factors (ADRFs), which are currently referred to as PVAT-derived relaxing factors (PVRFs), play a critical role; however, the mechanism is unclear. Ang 1 to 7 may be potential candidates of PVRFs given that Ang 1 to 7 act on the endothelium to cause the release of nitric oxide, which acts as a hyperpolarizing factor through $\mathrm{K}(\mathrm{Ca})$ channels to cause relaxation of the blood vessel in rat aorta [56]. Whether others factors, such as adiponectin, leptin, hydrogen sulfate $\left(\mathrm{H}_{2} \mathrm{~S}\right)$ generated by cystathionine gamma lyase (CSE), and palmitic acid methyl ester (PAME), are PVRF members remains controversial. Taken together, these results all highlight the proatherogrnic role of PVAT in the development of atherosclerosis based on various studies about the antiatherogenic effect of PVAT $[57,58]$.

\section{Conclusions}

Conclusively, the functional significance of the inflammatory response in atherosclerosis is increasingly conspicuous. However, the complicated pathogenesis of atherosclerosis remains unclear. From our perspective, endothelial dysfunction, SMC migration and proliferation, the transformation of fibroblasts into myofibroblasts, and adipokines produced by PVAT are predominantly implicated in the pathological process of atherosclerosis. We are looking forward to the discovery of positive and effective therapeutic approaches to reduce the incidence and mortality and ameliorate the prognosis of atherosclerosis-related disorders via restoring the normal function of the endothelium or inhibiting the above processes. Of course, in view of some controversial ideas, we should further investigate the possible roles of different cells from the arterial vessel wall in atherosclerosis to provide a solid foundation for therapeutic interventions for atherosclerosis and its associated disorders.

\section{Abbreviations}

PVAT: Perivascular adipose tissue

SMCs: $\quad$ Smooth muscle cells

ECs: $\quad$ Endothelial cells

NO: $\quad$ Nitric oxide

$\mathrm{PGI}_{2:} \quad$ Prostaglandin $\mathrm{I}_{2}$

EDHF: Endothelium-derived hyperpolarizing factor

EDRFs: Endothelium-derived relaxing factors

ET-1: $\quad$ Endothelin 1

TXA $_{2}$ : Thromboxane $\mathrm{A}_{2}$

Ang II: Angiotensin II

$\mathrm{UP}_{4} \mathrm{~A}$ : $\quad$ Uridine adenosine tetraphosphate

EDCFs: Endothelium-derived contracting factors

ED: $\quad$ Endothelial dysfunction

ROS: $\quad$ Reactive oxygen species

TNF- $\alpha$ : Tumor necrosis factor $\alpha$

IL: Interleukin

VCAM: Vascular cell adhesion molecule

MCP-1: $\quad$ Monocyte chemoattractant protein 1 


$\begin{array}{ll}\text { SR: } & \text { Scavenger receptor } \\ \text { FCs: } & \text { Foam cells } \\ \text { vWF: } & \text { von Willebrand factor } \\ \text { SM-MHC: } & \text { Smooth muscle myosin heavy chain } \\ \alpha \text { SMA: } & \alpha \text {-Smooth muscle-actin } \\ \text { ECMs: } & \text { Extracellular matrices } \\ \text { LDLR: } & \text { Low-density lipoprotein receptor } \\ \text { PDGF: } & \text { Platelet-derived growth factor } \\ \text { TGF- } \beta: & \text { Transforming growth factor } \beta \\ \text { MIF: } & \text { Macrophage inhibitory factor } \\ \text { IFN- } \gamma: & \text { Interferon } \gamma \\ \text { GFs: } & \text { Growth factors } \\ \text { NADPH: } & \text { Nicotinamide adenine dinucleotide phosphate } \\ & \text { hydrate } \\ \text { AFs: } & \text { Activated fibroblasts } \\ \text { TRPV1: } & \text { Transient receptor potential vanilloid type 1 } \\ \text { Th1: } & \text { T helper } 1 \\ \text { Treg: } & \text { Regulatory T } \\ \text { NKT: } & \text { Natural killer T } \\ \text { DCs: } & \text { Dendritic cells } \\ \text { IRA: } & \text { Innate responsive activator } \\ \text { GM-CSF: } & \text { Granulocyte-macrophage colony-stimulating } \\ & \text { factor } \\ \text { FFAs: } & \text { Free fatty acids } \\ \text { PAI-1: } & \text { Plasminogen activator inhibitor 1 } \\ \text { EAT: } & \text { Empirical adipose tissue } \\ \text { SAT: } & \text { Subcutaneous adipose tissue } \\ \text { ADRFs: } & \text { Adventitium-derived relaxing factors } \\ \text { PVRFs: } & \text { PVAT-derived relaxing factors } \\ \text { H } 2 \text { S: } & \text { Hydrogen sulfate } \\ \text { CSE: } & \text { Cystathionine gamma lyase } \\ \text { PAME: } & \text { Palmitic acid methyl ester. } \\ & \end{array}$

\section{Conflicts of Interest}

The authors declare that they have no conflicts of interest.

\section{Acknowledgments}

Our work was supported by 81470471 from the Nature Science Foundation of China (NSFC).

\section{References}

[1] A. D. Lopez, C. D. Mathers, M. Ezzati, D. T. Jamison, and C. J. Murray, "Global and regional burden of disease and risk factors, 2001: systematic analysis of population health data," Lancet, vol. 367, no. 9524, pp. 1747-1757, 2006.

[2] D. Mozaffarian, E. J. Benjamin, A. S. Go et al., "Heart disease and stroke statistics-2016 update: a report from the American Heart Association," Circulation, vol. 133, no. 4, pp. e38-360, 2016.

[3] R. Ross, "The pathogenesis of atherosclerosis: a perspective for the 1990s," Nature, vol. 362, no. 6423, pp. 801-809, 1993.

[4] Y. Nakashima, H. Fujii, S. Sumiyoshi, T. N. Wight, and K. Sueishi, "Early human atherosclerosis: accumulation of lipid and proteoglycans in intimal thickenings followed by macrophage infiltration," Arteriosclerosis, Thrombosis, and Vascular Biology, vol. 27, no. 5, pp. 1159-1165, 2007.
[5] I. Tabas, K. J. Williams, and J. Boren, "Subendothelial lipoprotein retention as the initiating process in atherosclerosis: update and therapeutic implications," Circulation, vol. 116, no. 16, pp. 1832-1844, 2007.

[6] M. L. Ballinger, N. Osman, K. Hashimura et al., "Imatinib inhibits vascular smooth muscle proteoglycan synthesis and reduces LDL binding in vitro and aortic lipid deposition in vivo," Journal of Cellular and Molecular Medicine, vol. 14, no. 6B, pp. 1408-1418, 2010.

[7] R. Ross, "Atherosclerosis-an inflammatory disease," The New England Journal of Medicine, vol. 340, no. 2, pp. 115-126, 1999.

[8] P. Libby, "Inflammation in atherosclerosis," Nature, vol. 420, no. 6917, pp. 868-874, 2002.

[9] C. Lahoute, O. Herbin, Z. Mallat, and A. Tedgui, "Adaptive immunity in atherosclerosis: mechanisms and future therapeutic targets," Nature Reviews. Cardiology, vol. 8, no. 6, pp. 348-358, 2011.

[10] U. Sadat, F. A. Jaffer, M. A. van Zandvoort, S. J. Nicholls, D. Ribatti, and J. H. Gillard, "Inflammation and neovascularization intertwined in atherosclerosis: imaging of structural and molecular imaging targets," Circulation, vol. 130, no. 9, pp. 786-794, 2014.

[11] K. Maiellaro and W. R. Taylor, "The role of the adventitia in vascular inflammation," Cardiovascular Research, vol. 75, no. 4, pp. 640-648, 2007.

[12] S. N. Verhagen and F. L. Visseren, "Perivascular adipose tissue as a cause of atherosclerosis," Atherosclerosis, vol. 214, no. 1, pp. 3-10, 2011.

[13] R. Ross and J. A. Glomset, "Atherosclerosis and the arterial smooth muscle cell: proliferation of smooth muscle is a key event in the genesis of the lesions of atherosclerosis," Science, vol. 180, no. 4093, pp. 1332-1339, 1973.

[14] J. Zhou, Y. S. Li, and S. Chien, "Shear stress-initiated signaling and its regulation of endothelial function," Arteriosclerosis, Thrombosis, and Vascular Biology, vol. 34, no. 10, pp. 21912198, 2014.

[15] P. R. Moreno, K. R. Purushothaman, E. Zias, J. Sanz, and V. Fuster, "Neovascularization in human atherosclerosis," Circulation, vol. 113, no. 18, pp. 2245-2252, 2006.

[16] T. Szasz and R. C. Webb, "Perivascular adipose tissue: more than just structural support," Clinical Science (London, England), vol. 122, no. 1, pp. 11-12, 2012.

[17] B. G. Schwartz, C. Economides, G. S. Mayeda, S. Burstein, and R. A. Kloner, "The endothelial cell in health and disease: its function, dysfunction, measurement and therapy," International Journal of Impotence Research, vol. 22, no. 2, pp. 77-90, 2010.

[18] X. Hu, X. Xu, G. Zhu et al., "Vascular endothelial-specific dimethylarginine dimethylaminohydrolase-1-deficient mice reveal that vascular endothelium plays an important role in removing asymmetric dimethylarginine," Circulation, vol. 120, no. 22, pp. 2222-2229, 2009.

[19] N. M. Hamburg, M. J. Keyes, M. G. Larson et al., "Cross-sectional relations of digital vascular function to cardiovascular risk factors in the Framingham Heart Study," Circulation, vol. 117, no. 19, pp. 2467-2474, 2008.

[20] K. H. Park and W. J. Park, "Endothelial dysfunction: clinical implications in cardiovascular disease and therapeutic approaches," Journal of Korean Medical Science, vol. 30, no. 9, pp. 1213-1225, 2015. 
[21] M. Barton, "Prevention and endothelial therapy of coronary artery disease," Current Opinion in Pharmacology, vol. 13, no. 2, pp. 226-241, 2013.

[22] A. Zernecke and C. Weber, "Chemokines in the vascular inflammatory response of atherosclerosis," Cardiovascular Research, vol. 86, no. 2, pp. 192-201, 2010.

[23] N. D. Brunetti, G. Salvemini, A. Cuculo et al., "Coronary artery ectasia is related to coronary slow flow and inflammatory activation," Atherosclerosis, vol. 233, no. 2, pp. 636-640, 2014.

[24] G. Luc, J. M. Bard, I. Juhan-Vague et al., "C-reactive protein, interleukin-6, and fibrinogen as predictors of coronary heart disease: the PRIME study," Arteriosclerosis, Thrombosis, and Vascular Biology, vol. 23, no. 7, pp. 1255-1261, 2003.

[25] J. E. Van Loon, M. Kavousi, F. W. Leebeek et al., "von Willebrand factor plasma levels, genetic variations and coronary heart disease in an older population," Journal of Thrombosis and Haemostasis, vol. 10, no. 7, pp. 1262-1269, 2012.

[26] G. K. Owens, "Regulation of differentiation of vascular smooth muscle cells," Physiological Reviews, vol. 75, no. 3, pp. 487-517, 1995.

[27] A. Margariti, L. Zeng, and Q. Xu, "Stem cells, vascular smooth muscle cells and atherosclerosis," Histology and Histopathology, vol. 21, no. 9, pp. 979-985, 2006.

[28] X. Z. Ruan, J. F. Moorhead, J. L. Tao et al., "Mechanisms of dysregulation of low-density lipoprotein receptor expression in vascular smooth muscle cells by inflammatory cytokines," Arteriosclerosis, Thrombosis, and Vascular Biology, vol. 26, no. 5, pp. 1150-5, 2006.

[29] Y. Ishikawa, M. Kimura-Matsumoto, M. Murakami et al., "Distribution of smooth muscle cells and macrophages expressing scavenger receptor BI/II in atherosclerosis," Journal of Atherosclerosis and Thrombosis, vol. 16, no. 6, pp. 829-839, 2009.

[30] J. Y. Jeremy, D. Rowe, A. M. Emsley, and A. C. Newby, "Nitric oxide and the proliferation of vascular smooth muscle cells," Cardiovascular Research, vol. 43, no. 3, pp. 580-594, 1999.

[31] J. Hou, X. Xue, and J. Li, "Vasostatin-2 inhibits cell proliferation and adhesion in vascular smooth muscle cells, which are associated with the progression of atherosclerosis," Biochemical and Biophysical Research Communications, vol. 469, no. 4, pp. 948-953, 2016.

[32] Y. Lang, D. Chen, D. Li et al., "Luteolin inhibited hydrogen peroxide-induced vascular smooth muscle cells proliferation and migration by suppressing the Src and Akt signalling pathways," The Journal of Pharmacy and Pharmacology, vol. 64, no. 4, pp. 597-603, 2012.

[33] S. M. Schwartz, R. Virmani, and M. E. Rosenfeld, "The good smooth muscle cells in atherosclerosis," Current Atherosclerosis Reports, vol. 2, no. 5, pp. 422-429, 2000.

[34] L. S. Shankman, D. Gomez, O. A. Cherepanova et al., "KLF4dependent phenotypic modulation of smooth muscle cells has a key role in atherosclerotic plaque pathogenesis," Nature Medicine, vol. 21, no. 6, pp. 628-637, 2015.

[35] A. C. Doran, N. Meller, and C. A. McNamara, "Role of smooth muscle cells in the initiation and early progression of atherosclerosis," Arteriosclerosis, Thrombosis, and Vascular Biology, vol. 28, no. 5, pp. 812-819, 2008.

[36] F. E. Rey, "The reactive adventitia: fibroblast oxidase in vascular function," Arteriosclerosis, Thrombosis, and Vascular Biology, vol. 22, no. 12, pp. 1962-1971, 2002.
[37] C. J. Schwartz and J. R. Mitchell, "Cellular infiltration of the human arterial adventitia associated with atheromatous plaques," Circulation, vol. 26, no. 1, pp. 73-78, 1962.

[38] Y. Shi, J. E. O'Brien, A. Fard, and A. Zalewski, “Transforming growth factor-beta 1 expression and myofibroblast formation during arterial repair," Arteriosclerosis, Thrombosis, and Vascular Biology, vol. 16, no. 10, pp. 1298-1305, 1996.

[39] M. Fujiwara, Y. Muragaki, and A. Ooshima, "Upregulation of transforming growth factor-beta1 and vascular endothelial growth factor in cultured keloid fibroblasts: relevance to angiogenic activity," Archives of Dermatological Research, vol. 297, no. 4, pp. 161-169, 2005.

[40] A. Jabs, E. I. Okamoto, J. Vinten-Johansen, G. Bauriedel, and J. N. Wilcox, "Sequential patterns of chemokine- and chemokine receptor-synthesis following vessel wall injury in porcine coronary arteries," Atherosclerosis, vol. 192, no. 1, pp. 75-84, 2007.

[41] F. Xu, J. Ji, L. Li, R. Chen, and W. C. Hu, “Adventitial fibroblasts are activated in the early stages of atherosclerosis in the apolipoprotein E knockout mouse," Biochemical and Biophysical Research Communications, vol. 352, no. 3, pp. 681-688, 2007.

[42] M. J. Haurani and P. J. Pagano, "Adventitial fibroblast reactive oxygen species as autacrine and paracrine mediators of remodeling: bellwether for vascular disease?" Cardiovascular Research, vol. 75, no. 4, pp. 679-689, 2007.

[43] J. Liu, A. Ormsby, N. Oja-Tebbe, and P. Pagano, "Gene transfer of $\mathrm{NAD}(\mathrm{P}) \mathrm{H}$ oxidase inhibitor to the vascular adventitia attenuates medial smooth muscle hypertrophy," Circulation Research, vol. 95, no. 6, pp. 587-594, 2004.

[44] F. Xu, Y. Liu, L. Shi et al., "NADPH oxidase p47phox siRNA attenuates adventitial fibroblasts proliferation and migration in apoE(-/-) mouse," Journal of Translational Medicine, vol. 13, no. 1, p. 38, 2015.

[45] M. J. Mulligan-Kehoe and M. Simons, "Vasa vasorum in normal and diseased arteries," Circulation, vol. 129, no. 24, pp. 2557-2566, 2014.

[46] C. L. Hu, J. Z. Xiang, and F. F. Hu, "Vanilloid receptor TRPV1, sensory C-fibers, and activation of adventitial mast cells. A novel mechanism involved in adventitial inflammation," Medical Hypotheses, vol. 71, no. 1, pp. 102-103, 2008.

[47] M. P. Moos, N. John, R. Gräbner et al., “The lamina adventitia is the major site of immune cell accumulation in standard chow-fed apolipoprotein E-deficient mice," Arteriosclerosis, Thrombosis, and Vascular Biology, vol. 25, no. 11, pp. 23862391, 2005.

[48] H. Ait-Oufella, A. P. Sage, Z. Mallat, and A. Tedgui, "Adaptive ( $\mathrm{T}$ and B cells) immunity and control by dendritic cells in atherosclerosis," Circulation Research, vol. 114, no. 10, pp. 1640$1660,2014$.

[49] C. Y. Miao and Z. Y. Li, "The role of perivascular adipose tissue in vascular smooth muscle cell growth," British Journal of Pharmacology, vol. 165, no. 3, pp. 643-658, 2012.

[50] M. Takaoka, H. Suzuki, S. Shioda et al., "Endovascular injury induces rapid phenotypic changes in perivascular adipose tissue," Arteriosclerosis, Thrombosis, and Vascular Biology, vol. 30, no. 8, pp. 1576-1582, 2010.

[51] M. R. Schroeter, N. Eschholz, S. Herzberg et al., "Leptindependent and leptin-independent paracrine effects of perivascular adipose tissue on neointima formation," Arteriosclerosis, Thrombosis, and Vascular Biology, vol. 33, no. 5, pp. 980-987, 2013. 
[52] A. Salgado-Somoza, E. Teijeira-Fernández, Á. L. Fernández, J. R. González-Juanatey, and S. Eiras, "Proteomic analysis of epicardial and subcutaneous adipose tissue reveals differences in proteins involved in oxidative stress," American Journal of Physiology. Heart and Circulatory Physiology, vol. 299, no. 1, pp. H202-H209, 2010.

[53] N. Maenhaut and J. Van de Voorde, "Regulation of vascular tone by adipocytes," BMC Medicine, vol. 9, no. 1, p. 25, 2011.

[54] D. Lamers, R. Schlich, S. Greulich, S. Sasson, H. Sell, and J. Eckel, "Oleic acid and adipokines synergize in inducing proliferation and inflammatory signalling in human vascular smooth muscle cells," Journal of Cellular and Molecular Medicine, vol. 15, no. 5, pp. 1177-1188, 2011.

[55] M. K. Öhman, W. Luo, H. Wang et al., "Perivascular visceral adipose tissue induces atherosclerosis in apolipoprotein E deficient mice," Atherosclerosis, vol. 219, no. 1, pp. 33-39, 2011.

[56] R. M. Lee, C. Lu, L. Y. Su, and Y. J. Gao, "Endothelium-dependent relaxation factor released by perivascular adipose tissue," Journal of Hypertension, vol. 27, no. 4, pp. 782-790, 2009.

[57] T. P. Fitzgibbons, S. Kogan, M. Aouadi, G. M. Hendricks, J. Straubhaar, and M. P. Czech, "Similarity of mouse perivascular and brown adipose tissues and their resistance to dietinduced inflammation," American Journal of Physiology. Heart and Circulatory Physiology, vol. 301, no. 4, pp. H1425-H1437, 2011.

[58] L. Chang, L. Villacorta, R. Li et al., "Loss of perivascular adipose tissue on peroxisome proliferator-activated receptorgamma deletion in smooth muscle cells impairs intravascular thermoregulation and enhances atherosclerosis," Circulation, vol. 126, no. 9, pp. 1067-1078, 2012. 


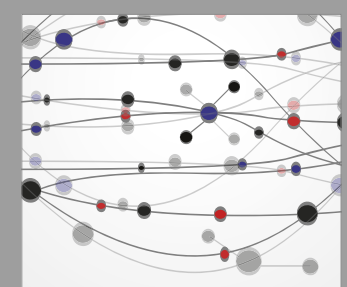

The Scientific World Journal
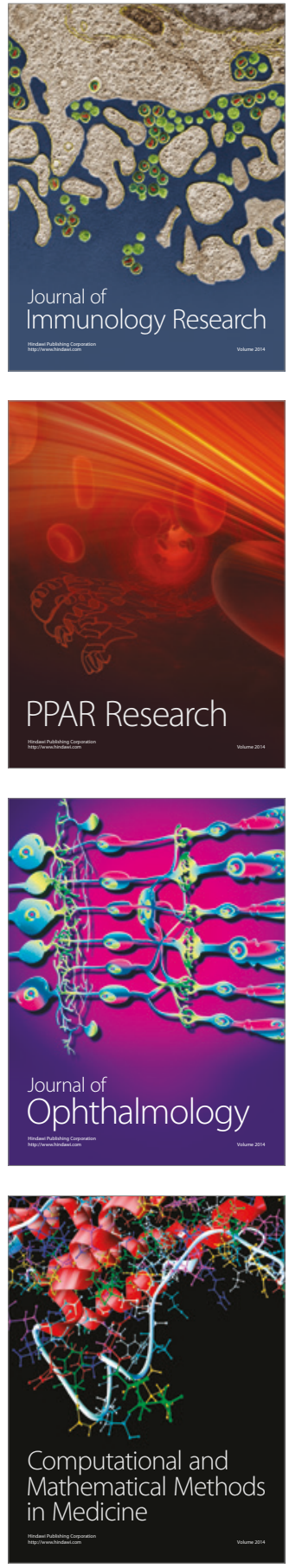

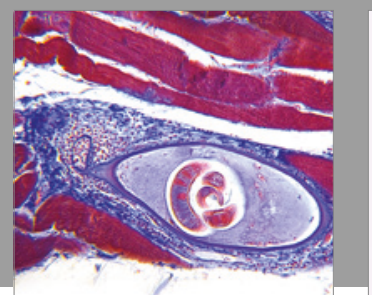

Gastroenterology Research and Practice
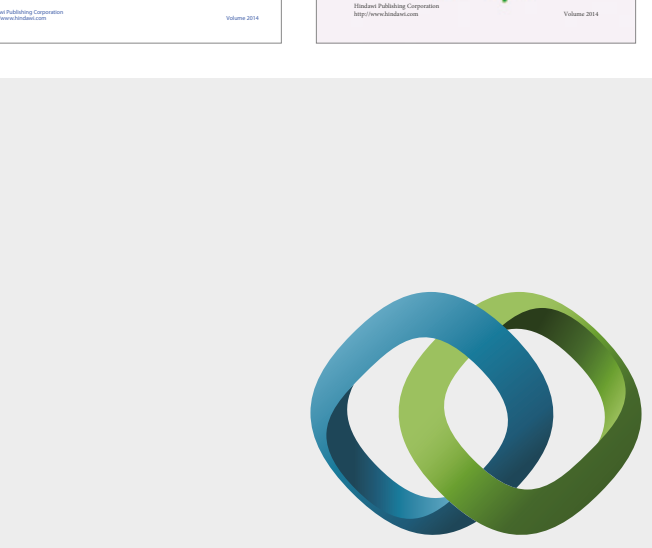

\section{Hindawi}

Submit your manuscripts at

https://www.hindawi.com
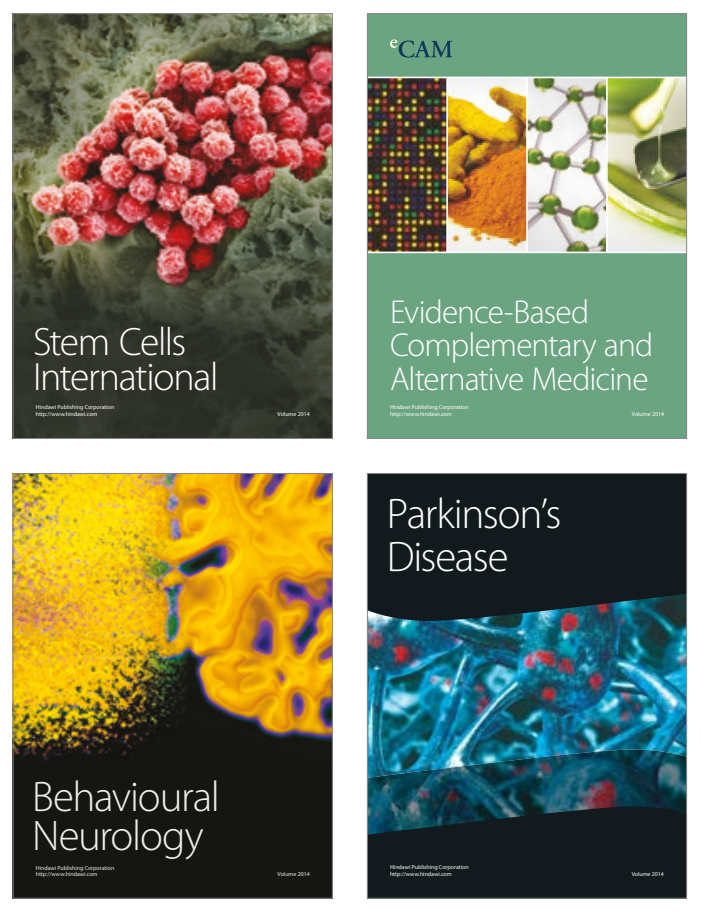
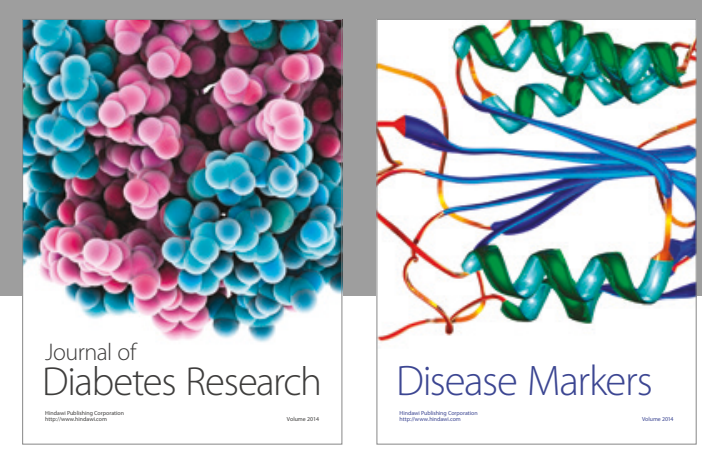

Disease Markers
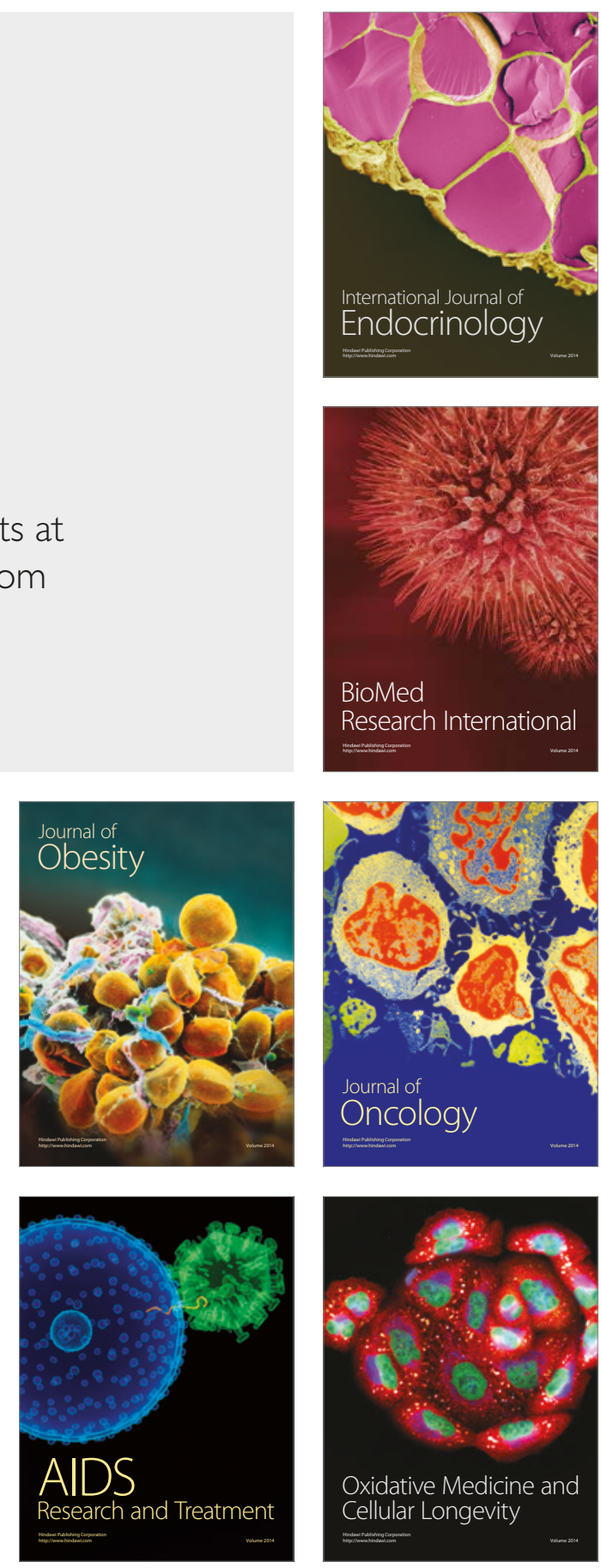